\title{
Existence of the Greek Letter Elements in the Stable Homotopy Groups of $E(n)_{*}$-Localized Spheres
}

\author{
By
}

Katsumi Shimomura* and Masaaki Yokotani**

\begin{abstract}
Let $E_{2}^{* * *}(X)$ denote the $E_{2}$-term of the Adams-Novikov spectral sequence converging to the homotopy groups of $X$. We can define the Greek letter elements in $E_{2}^{* * *}\left(L_{n} S^{0}\right)$ in the same way as those in $E_{2}^{* * *}\left(S^{0}\right)$. We show that these of $E_{2}^{*, *}\left(L_{n} S^{0}\right)$ are permanent cycles, though we know little for $E_{2}^{*, *}\left(S^{0}\right)$.
\end{abstract}

\section{$\S 1$. Introduction}

Let $B P$ denote the Brown-Peterson spectrum whose homotopy groups are $Z_{(p)}\left[v_{1}, v_{2}, \cdots\right]$ over the Hazewinkel generators $v_{n}$ 's. By the insight of Toda's construction of $V(3)[\mathrm{T}], \mathrm{Z}$. Yosimura and the first named author constructed a $B P$ version of Toda's construction [SY]. In [SY], it is studied the $L_{n}$-local spectrum $Y J$ whose $B P_{*}$-homology is $v_{n}^{-1} B P_{*} / W(J)$ for an invariant regular sequence of length only $n$. Here $n$ is a positive integer and $L_{n}$ is the Bousfield localization with respect to the spectrum $v_{n}^{-1} B P$, which is the same one with respect to the Johnson-Wilson spectrum $E(n)$. Here $\pi_{*}(E(n))=\boldsymbol{Z}_{(p)}\left[v_{1}, \cdots, v_{n}, v_{n}^{-1}\right]$.

In this paper, we show that [SY] is applied for the case that the length of the sequence $J$ is less than $n$, and obtain

Theorem 1.1. Let $n$ and $m$ be integers such that $0 \leq m \leq n$ and $n^{2}+n<2 p$. Then there exists a spectrum YJ such that

Communicated by K. Saito, December 28, 1992.

1991 Mathematics Subject Classification: Primary 55N22, 55Q45, Secondary 55N20, 55P42, 55Q10, 57R77.

Key Words: BP-Theory, Stable Homotopy of Spheres.

* Department of Mathematics, Faculty of Education, Tottori University, Tottori 680, Japan.

** Graduate School of Science and Technology, Okayama University, Okayama 700, Japan. 


$$
E(n)_{*}(Y J) \cong E(n)_{*} /\left(J_{m}\right)
$$

as $E(n)_{*}$-module.

F. Adams introduced the first Greek letter elements in the stable homotopy groups of spheres, and then the second and the third Greek letter elements were defined by $\mathrm{H}$. Toda, L. Smith, S. Oka and so on. In their paper [MRW], H. Miller, D. Ravenel and S. Wilson defined the general Greek letter elements in the $E_{2}$-term of the Adams-Novikov spectral sequence based on $B P$ converging to the stable homotopy groups of spheres. For the Greek letter elements given earlier in the stable homotopy, the corresponding elements in the $E_{2}$-term agree with the newly defined Greek letter elements in the $E_{2}$-term. Toda and et al's Greek letter elements are based on the spectrum $X J$ such that $B P_{*}(X J)=B P_{*} /(J)$.

In most cases, it is still unknown whether or not those Greek letter elements in the $E_{2}$-term survive to $E_{\infty}$, though we know that some of each $n$-th Greek letter elements are permanent cycles by a result of [HS].

Here we show that the Greek letter elements are all permanent cycles after replacing the sphere spectrum by the $L_{n}$-localized spheres under some condition on $n$. We define the Greek letter elements $g\left(J_{m}\right)$ in the $E_{2}$-term for an invariant regular sequence $J_{m}$ with $m \leq n$ by the image of a composition of connecting homomorphisms associated to $J_{m}$ (see (5.1)). We denote the corresponding homotopy element by $\alpha\left(J_{m}\right)$ (see (5.4)). As Theorem 1.1, we have an analogous spectrum $Y J$ to $X J$ if we replace $B P$ by $E(n)$. So in the same way as the construction of Toda and et al's Greek letter elements, we obtain

Theorem 1.2. Let $n$ and $m$ be non-negative integers with $m \leq n$ and $n^{2}+n \leq 2 p$. Then we have the homotopy element $\alpha\left(J_{m}\right)$ in $\pi_{*}\left(L_{n} S^{0}\right)$.

We prove Theorem 1.1 in $\S 4$ by a similar fashion to that of [SY]. In other words, we construct a $B P$-based Adams tower from the $E_{1}$-term, which is shown to give an Adams-type spectral sequence converging to the homotopy groups of the $E(n)$-localized spectrum by $\mathrm{M}$. Hopkins and $\mathbb{D}$. Ravenel, recently. Unhappily, $B P_{*}\left(L_{n} X\right) \neq v_{n}^{-1} B P_{*}(X)$ for general $X$, and we cannot apply the theorem of $[\mathrm{SY}]$ without any change.

This paper is organized as follows: In $\S 2$, we study the ring structure of a telescope of a ring spectrum related to $B P$. We adjust the results of [SY] 
to fit to our case in $\S 3$ and prove Theorem 1.1 and Theorem 1.2 in $\S 4$ and $\S 5$, respectively.

\section{§2. BP-Hopf Module Spectra $v_{n}^{-1} B P J$}

Let $E$ be a ring spectrum and $A$ an $E$-module spectrum with structure map $\phi: E \wedge A \rightarrow A$. We call a spectrum $A$ E-quasi associative ring spectrum ([JY]), if there is a multiplication $\mu: A \wedge A \rightarrow A$ satisfying the following three yconditions:

$$
\begin{gathered}
\mu(\phi \wedge 1)=\phi(1 \wedge \mu): E \wedge A \wedge A \rightarrow A, \\
\mu(\phi \wedge 1)(T \wedge 1)=\mu(1 \wedge \phi): A \wedge E \wedge A \rightarrow A, \text { and } \\
\mu(1 \wedge \phi)(1 \wedge T)=\phi T(\mu \wedge 1): A \wedge A \wedge E \rightarrow A .
\end{gathered}
$$

We fix a prime number $p$ and consider the Brown-Peterson spectrum $B P$. $\quad B P$ is a ring spectrum, whose homotopy and $B P_{*}$-homology groups are

$$
\begin{aligned}
& B P_{*}=\pi_{*}(B P)=\mathbb{Z}_{(p)}\left[v_{1}, v_{2}, \cdots\right], \text { and } \\
& B P_{*}(B P)=\pi_{*}(B P \wedge B P)=B P_{*}\left[t_{1}, t_{2}, \cdots\right],
\end{aligned}
$$

where the degrees of the generators are $\operatorname{deg} v_{n}=\operatorname{deg} t_{n}=2 p^{n}-2$, which we denote $q_{n}$. We denote the unit map and the multiplication of $B P$ by $i: S^{0} \rightarrow B P$ and $\mu: B P \wedge B P \rightarrow B P$, respectively. We also denote $\overline{B P}$ for the cofiber of the unit map $i$.

Throughout this paper we fix a positive integer

$n$.

Let $A$ be a $B P$-module spectrum with structure map $v: B P \wedge A \rightarrow A$. Then we have a self map $\dot{v}_{n}: A \rightarrow \Sigma^{-q_{n}} A$ as in the above theorem induced from the element $v_{n} \in B P_{*}$ whose degree is $q_{n}=2 p^{n}-2$. Suppose that any composition of $\dot{v}_{n}$ is not trivial. We then have the homotopy colimit (telescope) $T A=\varliminf_{i} \Sigma^{-i q_{n}} A$, and $\pi_{*}(T A)=v_{n}^{-1} \pi_{*}(A)$. We consider the following condition:

$A$ consists of cells $e_{\alpha}$ such that $\operatorname{deg} e_{\alpha} \equiv \mathrm{r} \bmod 2 p-2$ with $0 \leq r \leq 2 p-4$.

We have the next lemma whose proof is due to T. Ohkawa. 
Lemma 2.2. Let $A$ be a BP-module spectrum and $B$ a spectrum. Suppose that $\pi_{r}(B)=0$ unless $r \equiv 0 \bmod 2 p-2$, and $A$ satisfies the condition (2.1). Then we have an isomorphism

$$
[T A, B] \cong \varliminf \varliminf[A, B]
$$

Proof. Consider the $i q_{n}$-skeleton $A^{(i)}$ of $A$. Then we obtain a map $\left(v_{n}\right)^{(i)}: A^{(i)} \rightarrow \Sigma^{-q_{n}} A^{(i+1)}$ induced from the map $\dot{v}_{n}: A \rightarrow \Sigma^{-q_{n}} A$. In other words, it satisfies that $\dot{v}_{n} \imath_{i}=\imath_{i+1}\left(v_{n}\right)^{(i)}$ for the inclusion $\imath_{j}: A^{(j)} \rightarrow A$. Let $T^{\prime} A$ denote the homotopy colimit of the maps $\left(v_{n}\right)^{(i)}$. Then we have a map $\imath$ : $T^{\prime} A \rightarrow T A$ given by the inclusions $\imath_{j}$, which induces an isomorphism $\imath_{*}: \pi_{*}\left(T^{\prime} A\right) \rightarrow \pi_{*}(T A)$ at each dimension. Thus the map $\imath$ is a homotopy equivalence.

We have Milnor's exact sequence

$$
0 \rightarrow \varliminf^{1}\left[\Sigma^{1-i q_{n}} A^{(i)}, B\right] \rightarrow\left[T^{\prime} A, B\right] \stackrel{i^{\prime *}}{\rightarrow} \varliminf_{\operatorname{im}}\left[\Sigma^{-i q_{n}} A^{(i)}, B\right] \rightarrow 0
$$

We notice that the group $\left[\Sigma^{1-i q_{n}} A^{(i)}, B\right]$ is a subquotient of a direct sum of homotopy groups $\pi_{r}(B)$ with $\operatorname{deg} r \neq \equiv \bmod 2 p-2$ by the assumption on $A$. Thus we have $\left[\Sigma^{1-i q_{n}} A^{(i)}, B\right]=0$ by the assumption on $B$ and we see that the $\varliminf^{1}$ group is null. Therefore we have the commutative diagram

$$
\begin{aligned}
& \left.\left[T^{\prime} A, B\right] \stackrel{i^{\prime *}}{\longrightarrow} \varliminf^{-i q_{n}} A^{(i)}, B\right] \\
& { }^{*} \uparrow \quad \varliminf \imath_{i}^{*} \uparrow \\
& {[T A, B] \stackrel{i^{*}}{\longrightarrow} \varliminf^{\lim }\left[\Sigma^{-i q_{n}} A, B\right]}
\end{aligned}
$$

in which $i^{*}$ and $\imath^{*}$ are isomorphisms. So $i^{*}$ is a monomorphism. On the other hand, it is an epimorphism by the Milnor's exact sequence on $T A$.

q.e.d.

Let $A$ be a $B P$-module spectrum with structure map $v: B P \wedge A \rightarrow A$. We call $A B P$-Hopf module spectrum if it is provided with $B P$-module map $\eta: A \rightarrow B P \wedge A$ such that $v \eta=1$ and $(1 \wedge \eta) \eta=(1 \wedge i \wedge 1) \eta$ for the identity 
map 1 ( $c f .[\mathrm{SY}])$.

It is known ( $c f .[\mathrm{R} 2])$ that the pair $\left(B P_{*}, B P_{*}(B P)\right)$ is a Hopf algebroid with structure maps: the right and the left units $\eta_{R}, \eta_{L}: B P_{*} \rightarrow B P_{*}(B P)$, the coproduct $\triangle: B P_{*}(B P) \rightarrow B P_{*}(B P) \bigotimes_{B P_{*}} B P_{*}(B P)$ and the counit $\varepsilon: B P_{*}(B P)$ $\rightarrow B P_{*}$, which are induced from $i \wedge 1,1 \wedge i: B P \rightarrow B P \wedge B P, \quad 1 \wedge i \wedge 1: B P$ $\wedge B P \rightarrow B P \wedge B P \wedge B P$ and $\mu: B P \wedge B P \rightarrow B P$, respectively. Let $J: a_{0}, a_{1}, \cdots$ be a sequence of $B P_{*}$ of finite length, and denote $J_{m}$ for a subsequence $a_{0}, a_{1}, \cdots, a_{m-1}$ of length $m . \quad\left(J_{m}\right)$ denotes the ideal of $B P_{*}$ generated by each entry $a_{k}$ of the sequence $J_{m}$, and especially, we put $\left(J_{0}\right)=0$. We call the sequence $J_{m}$ invariant if $\eta_{R}\left(a_{k}\right) \equiv a_{k} \bmod \left(J_{k}\right)$ for $0 \leq k<m$, and regular if the multiplication by $a_{k}: B P_{*} /\left(J_{k}\right) \rightarrow B P_{*} /\left(J_{k}\right)$ is a monomorphism for $0 \leq k<m$.

Let $J: a_{0}, a_{1}, \cdots, a_{n-1}$ be an invariant regular sequence of $B P_{*}$ of length $n$. Then there exists an $B P$-module spectrum $B P J$ with structure $v_{J}: B P \wedge B P J \rightarrow B P J$, whose homotopy groups are given by $\pi_{*}(B P J)=B P_{*} /(J)$ ([B]). For subsequences $J_{m}$ of $J$, these $B P J_{m}$ 's fit into the cofiber sequence

$$
\Sigma^{\operatorname{deg} a_{m}} B P J_{m} \stackrel{\dot{a}_{m}}{\rightarrow} B P J_{m} \stackrel{j_{m}}{\rightarrow} B P J_{m+1} \stackrel{k_{m}}{\rightarrow} \Sigma^{\operatorname{deg} a_{m}+1} B P J_{m},
$$

where $\dot{a}_{m}$ denotes the composition $v_{J_{m}}\left(a_{m} \wedge 1\right)$ for $a_{m} \in \pi_{*}(B P)$. Furthermore, it is a $B P$-quasi associative ring spectrum and also is a $B P$-Hopf module spectrum if $n<2 p-2$, by [SY; Proposition 1.2].

By $[\mathrm{BP}](c f .[\mathrm{P}]), B P$ consists of cells of degree $0 \bmod 2 p-2$. So using the cofiber sequences (2.3) inductively, we see that $B P J_{m}$ satisfies the condition (2.1) if $m \leq 2 p-4$. Put $A(m)=T B P J_{m}$ for $m \leq n$. Then we have the following from Lemma 2.2 , by an easy argument on limit and colimit.

Theorem 2.4. Let $m$ be a positive integer with $m \leq \min \{p-2, n\}$, and $J_{m}$ is a subsequence of an invariant regular sequence $J$ of length $n$. Then we have a BP-quasi associative ring spectrum $A(m)$ whose homotopy groups are $v_{n}^{-1} B P_{*} /\left(J_{m}\right)$, which is also a BP-Hopf module spectrum.

Corollary 2.5. For each integer $n, v_{n}^{-1} B P$ is a ring spectrum.

\section{§3. Geometric Resolution and $k$-Adams Tower}

First recall $[\mathrm{SY}]$ some facts and notations on $B P$-Hopf module spectra. Define the structure maps by $v=\mu \wedge 1$ and $\eta=1 \wedge i \wedge 1$, and we have a $B P$-Hopf module spectrum $B P \wedge X$ for any spectrum $X$. We call 
a $B P$-Hopf module spectrum $A$ an extended $B P$-Hopf module spectrum if $A$ is homotopic to $B P \wedge B$ for a $B P$-module spectrum $B$ as $B P$-Hopf module spectrum. Note that an extended $B P$-Hopf module spectrum $A$ gives rise to an extended $B P_{*}(B P)$-comodule $A_{*}(X)=\pi_{*}(A \wedge X)$ for any spectrum $X$. Consider a sequence

$$
\cdots \rightarrow A_{0} \stackrel{k_{0}}{\rightarrow} A_{1} \rightarrow \cdots \rightarrow A_{j} \stackrel{k_{j}}{\rightarrow} A_{j+1} \rightarrow \cdots
$$

We call the sequence a complex if the composition $k_{j} k_{j-1}$ is null for each $j$. Furthermore suppose that it is a sequence of $E$-module spectra for a ring spectrum $E$. We call it split if there exist $E$-module maps $s_{j}: A_{j+1} \rightarrow$ $A_{j}$ such that $s_{j} s_{j+1}=0$ and $k_{j} s_{j}+s_{j+1} k_{j+1}=1$.

Now recall [SY] the definition of geometric resolution and $k$-Adams tower. Let $E$ and $M$ be $B P$-Hopf module spectra. We call a complex $G=\left\{G_{j}, d_{j}: G_{j} \rightarrow G_{j+1}\right\}_{j \geq 0}$ an $\mathbb{E}$-geometric resolution over $M$ if it satisfies the following three conditions:

(i) There exists a $B P$-Hopf module map $\delta: M \rightarrow E \wedge G_{0}$ with $(1 \wedge$ $\left.d_{0}\right) \delta=0$.

(ii) The complex

$$
* \rightarrow M \stackrel{\delta}{\rightarrow} E \wedge G_{0} \stackrel{1 \wedge d 0}{\longrightarrow} E \wedge G_{1} \rightarrow \cdots \rightarrow E \wedge G_{k} \stackrel{1 \wedge d_{k}}{\longrightarrow} E \wedge G_{k+1} \rightarrow \cdots
$$

splits as a sequence of $B P$-module spectra.

(iii) The entry $E \wedge G_{k}$ of the complex is an extended $B P$-Hopf module spectrum for each $k \geq 0$.

Then we have

(3.1) [SY; Theorem 3.3] There exists a BP-geometric resolution $G=\left\{G_{k}\right.$ $\left.=\overline{B P}^{k} \wedge E, d_{k}: G_{k} \rightarrow G_{k+1}\right\}$ over a BP-Hopf module spectrum $E$.

Consider a set $X(k)=\left\{X_{j}, a_{j}, b_{j}, c_{j}\right\}_{1 \leq j \leq k}$ of spectra $X_{j}$ and maps $a_{j}: X_{j} \rightarrow \Sigma X_{j-1}, b_{j}: X_{j} \rightarrow W_{j+1}$ and $c_{j}: W_{j} \rightarrow X_{j}$, and an $E$-geometric resolution $G=\left\{G_{j}, d_{j}\right\}_{j \geq 0}$. Then we call $X(k) k$-Adams tower over $G$ if it satisfies the following properties:

(i) $X_{j-1} \stackrel{b_{j-1}}{\rightarrow} W_{j} \stackrel{c_{j}}{\rightarrow} X_{j} \stackrel{a_{j}}{\rightarrow} \sum X_{j-1}$ is a cofiber sequence, and

(ii) $d_{j-1}=b_{j-1} c_{j-1}$ and $d_{m} b_{m-1}=0$ for each $j$ with $1 \leq j \leq m$, where $X_{0}=W_{0}, b_{0}=d_{0}, c_{0}=1$ and $1 \leq m \leq \infty$. We note that $k$-Adams tower 
is called $k$-factorized system in [SY]. If there exists a spectrum $Y J$ such that $B P_{*}(Y J)=v_{n}^{-1} B P_{*} /(J)$, then the so-called $B P$-Adams tower for $Y J$ is an $\infty$-Adams tower in our sense. So this naming seems reasonable.

We call $B P J$-module spectrum $M$ quasi-associative if $\varphi_{M}\left(v_{J} \wedge \hat{1}\right)=$ $v_{M}\left(1 \wedge \varphi_{M}\right): \quad B P \wedge B P J \wedge M \rightarrow M$ and $v_{M}\left(1 \wedge \varphi_{M}\right)(T \wedge 1)=\varphi_{M}\left(1 \wedge v_{M}\right): B P J \wedge$ ${ }_{B P} \wedge M \rightarrow M$, where $\varphi_{M}$ denotes the $B P J$-module structure map and $v_{M}=\varphi_{M}(j \wedge 1)$ for the map $j: \mathbb{B} \rightarrow B P J$.

Let $F$ be a quasi-associative $B P J$-module spectrum and $G=\left\{G_{k}, d_{k}\right\}$ a $B P J$-geometric resolution over $M$. As we remarked in [SY], the Kronecker product $\kappa:\left[\Sigma^{t} G_{k}, F\right] \rightarrow \operatorname{Hom}_{B P J_{*}}^{t}\left(B P J_{*}\left(G_{k}\right), F_{*}\right)$ is an isomorphism for each $k \geq 0$ if $B P J_{*}\left(G_{k}\right)$ is $B P J_{*}$-free.

(3.2) [SY; Theorem 4.6] Suppose that the length of $J$ is less than $p-1$. Let $G=\left\{G_{k}, d_{k}\right\}_{k \geq 0}$ be a BP-geometric resolution over $M$ such that $M$ and $G_{k}$ for $k \geq 0$, are quasi-associative BPJ-module spectra with $\pi_{*}\left(G_{k}\right) B P J_{*}$-free and $\pi_{*}\left(G_{k}\right)=0$ unless $* \equiv 0 \bmod 2 p-2$. If $\operatorname{Ext}_{B P_{*}(B P)}^{m+2, m+t}\left(M_{*}, M_{*}\right)=0$ for all $m \geq 1$ and $t \in \mathbb{\Lambda}_{J}$, then $G$ adinits an $\infty$-Adams tower $X(\infty)$. Moreover, its $\infty$-Adams tower is uniquely given if $\operatorname{Ext}_{B P_{*}(B P)}^{m+1, m+t}\left(M_{*}, M_{*}\right)=0$ for all $m \geq 1$ and $t \in \Lambda_{J}$.

Here,

$$
\Lambda_{J}=\left\{\sum_{j} \varepsilon_{j} d_{j} \mid d_{j}=\operatorname{deg} a_{j}+1 \text { and } \varepsilon_{j} \in\{0,1\}\right\}
$$

for an invariant regular sequence $J: a_{0}, \cdots, a_{n-1}$.

By virtue of Lemma 2.2, the Kronecker product $\kappa$ stays an isomorphism even if we replace $G_{k}$ by $T G_{k}=\varliminf_{i_{n}} G_{k}$, under the assumption that $F$ satisfies (2.1). By taking the telescope $T$, we obtain a $B P$-geometric resolution $T G=\left\{T G_{k}=\overline{B P}^{k} \wedge T E, d_{k}: T G_{k} \rightarrow T G_{k+1}\right\}$ over a $B P-H o p f$ module spectrum $T E$ from (3.1). Now put $E=B P J_{m}$, and we see that it satisfies the condition of (3.2), and we rewrite (3.1-2) to fit to our case:

Theorem 3.3 Assume that $0 \leq m \leq n<p-1$. If $\operatorname{Ext}_{B P_{*}(B P)}^{k+2, k+t}\left(B P_{*}\right.$, $\left.v_{n}^{-1} B P_{*} /\left(J_{m}\right)\right)=0$ for all $k>0$ and $t \in \Lambda_{J_{\mathrm{m}}}$, then we have an $\infty$-Adams tower $X(\infty)$ over the BP-geometric resolution $T G=\left\{T G_{k}, d_{k}\right\}$ obtained from (3.2) over $v_{n}^{-1} B P J_{m}$. Furthermore, it is uniquely given if $\operatorname{Ext}_{\left.B P_{*}+B P\right)}^{k+1, k+t}\left(B P_{*}, v_{n}^{-1} B P_{*} /\left(J_{m}\right)\right)$ $=0$ for all $k>0$ and $t \in \Lambda_{J_{\mathrm{m}}}$.

Here the uniqueness follows from 
Theorem 3.4. Consider a map $f=\left\{f_{n}\right\}: G \rightarrow H$ of BP-geometric resolutions over $v_{n}^{-1} B P J$. If $\operatorname{Ext}_{B P_{*}(B P)}^{k+1, k+d+t}\left(B P_{*}, v_{n}^{-1} B P_{*} /\left(J_{m}\right)\right)=0$ for all $k>0$ and $t \in \Lambda_{J}$, then we have a map $\bar{f}=\left\{\bar{f}_{n}, f_{n}\right\}: X(\infty) \rightarrow Y(\infty)$ of $\infty$-Adams towers.

Proof. Recall [SY] that

(3.5) [SY; Proposition 4.3] Let $G=\left\{G_{k}, d_{k}\right\}_{k \geq 0}$ and $G^{\prime}=\left\{G_{k}^{\prime}, d_{k}^{\prime}\right\}_{k \geq 0}$ be E-geometric resolutions over $M$ and $N$, respectively, and $X(m)=\left\{X_{j}\right\}_{1 \leq j \leq m}$ and $X^{\prime}(m)=\left\{X_{j}^{\prime}\right\}_{1 \leq j \leq m}$ be their $m$-Adams towers. Given a map $g: G \rightarrow G^{\prime}$ of complexes, there exists a map $f(m): X(m) \rightarrow X^{\prime}(m)$ of $m$-Adams towers if $\left[\Sigma G_{k}, G_{k+2}^{\prime}\right]=0$ and the sequence $\left[\Sigma X_{k-1}, G_{k}^{\prime}\right] \rightarrow\left[\Sigma X_{k-1}, G_{k+1}^{\prime}\right] \rightarrow\left[\Sigma X_{k-1}, G_{k+2}^{\prime}\right]$ are exact for all $k$ with $1 \leq k<m$.

Then the theorem follows immediately from (3.5), since we see by Lemma 2.2 that the hypothesis of the theorem satisfy the conditions of (3.5).

q.e.d.

\section{§4. Spectrum whose $\boldsymbol{v}_{n}^{-1} B P_{*}$-Homology is $\boldsymbol{v}_{n}^{-1} \mathbb{B} P_{*} /\left(J_{m}\right)$}

For $m \leq n$, we have the change of rings theorem

$$
\operatorname{Ext}_{B P_{*}(B P)}^{s, t}\left(B P_{*}, v_{n}^{-1} B P_{*} /\left(J_{m}\right)\right)=\operatorname{Ext}_{E(n)_{*}(E(n))}^{s, t}\left(E(n)_{*}, E(n)_{*} /\left(J_{m}\right)\right),
$$

for the Johnson-Wilson spectrum $E(n)$ which has the coefficient ring $\mathbb{Z}_{(p)}\left[v_{1}, \cdots, v_{n}, v_{n}^{-1}\right] \subset v_{n}^{-1} B P_{*}$. The spectrum $E(n)$ is defined to be a spectrum that represents the homology theory $E(n)_{*}(X)=E(n)_{*} \otimes_{B P_{*}} B P_{*}(X)$. In [R1,(10.11)], Ravenel computed to show

$$
\operatorname{Ext}_{E(n)_{*}(E(n))}^{s, *}\left(E(n)_{*}, E(n)_{*} / I_{m}\right)=0
$$

for $s>n^{2}+n-m$ by using the chromatic spectral sequence. Now use the Filtration Theorem [JY; Theorem 1.16], and we have the following

Lemma 4.1. If $0 \leq m \leq n<p-1$, then

$$
\operatorname{Ext}_{B P_{*}(B P)}^{s, t}\left(B P_{*}, v_{n}^{-1} B P_{*} /\left(J_{m}\right)\right)=0
$$

for any integers $s$ with $s>n^{2}+n-m$ and $t$.

Lemma 4.2. Let $X(\infty)=\left(X_{j}, a_{j}, b_{j}, c_{j}\right)$ be an $\infty$-Adams tower over 
the geometric resolution $T G=\left\{T G_{k}, d_{k}\right\}$ over $v_{n}^{-1} B P J_{*}$ in Theorem 3.2. Then

$$
v_{n}^{-1} B P_{*}\left(\varliminf_{i} X_{i} \cong v_{n}^{-1} B P_{*} /(J)\right.
$$

as $B P_{*}$-module. See [R2; Definition 2.1.10] for a definition of $\varliminf X_{i}$.

Proof. Applying $B P_{*}$-homology to the tower $X(\infty)$, we obtain an exact couple, which gives an Adams-type spectral sequence. By the definition of geometric resolution, we see that the $E_{2}$-term is given by $E_{2}^{s . *}=0$ for $s>0$ and $E_{2}^{0, *}=v_{n}^{-1} B P_{*} /(J)$. Therefore this spectral sequence collapses and converges to $B P_{*}\left(\varliminf_{i} X_{i}\right)$.

q.e.d.

Now applying Lemmas $4.1-2$ to Theorem 3.3 , we get

Theorem 4.3. Let $J_{m}$ be an invariant regular sequence of length $m$. If $n^{2}+n<2 p$, then there exists a spectrum $Y J_{m}$ such that

$$
v_{n}^{-1} B P_{*}\left(Y J_{m}\right) \cong v_{n}^{-1} B P_{*} /\left(J_{m}\right)
$$

as $B P_{*}$-module.

\section{$\S 5$. The Greek Letter Elements}

Let $E(n)$ be the Johnson-Wilson spectrum with $E(n)_{*}=Z_{(p)}\left[v_{1}, \cdots, v_{n}, v_{n}^{-1}\right]$ and it gives rise to a homology theory $E(n)_{*}(X)=E(n)_{*} \otimes_{B P_{*}} B P_{*}(X)$ for any spectrum $X$. In the same way as [MRW], we define the Greek letter elements in the $E_{2}$-term of the Adams-Novikov spectral sequence computing $\pi_{*}\left(L_{n} S^{0}\right)$. Let $J_{m}: a_{0}, \cdots, a_{m-1}$ be an invariant regular sequence of $E(n)_{*}$

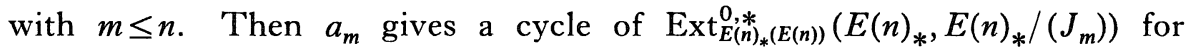
$J_{m}$. We use here an abbreviation: $H^{s . t} M=\operatorname{Ext}_{E(n)_{*}(E(n))}^{s, t}\left(E(n)_{*}, M\right)$ for an $E(n)_{*}(E(n))$-comodule $M$. Using this abbreviation, we have $a_{m} \in H^{0, *} E(n)_{*} /$ $\left(J_{m}\right)$. For each $k$, the short exact sequence $0 \rightarrow E(n)_{*} /\left(J_{k}\right) \stackrel{a_{k}}{\rightarrow} E(n)_{*} /\left(J_{k}\right)$ $\rightarrow E(n)_{*} /\left(J_{k+1}\right) \rightarrow 0$ gives the associated connecting homomorphism $\delta_{k}: H^{s, *} E(n)_{*} /\left(J_{k+1}\right) \rightarrow H^{s+1, *} E(n)_{*} /\left(J_{k}\right)$. Now define an $m$-th Greek letter element $g\left(J_{m}\right)$ associating to the sequence $J_{m}$ by

$$
g\left(J_{m}\right)=\delta_{0} \cdots \delta_{m-1}\left(a_{m}\right) \in H^{m, *} E(n)_{*} .
$$

We have shown that the existence of spectra $Y J_{m}$ such that 
$E(n)_{*}\left(Y J_{m}\right)=E(n)_{*} /\left(J_{m}\right)$ by Theorem 4.3 , since $E(n)_{*}\left(Y J_{m}\right)=\mathbb{E}(n)_{*} \otimes_{B P_{*}}$ $B P_{*}\left(Y J_{m}\right)$.

Lemma 5.2. Let $m$ be an integer with $0 \leq m \leq n$ and $n^{2}+n<2 p$. Then there exists a self map $\alpha_{m}: Y J_{m} \rightarrow Y J_{m}$ whose cofiber is $Y J_{m+1}$, and so $\mathbb{E}(n)_{*}\left(\alpha_{m}\right)=a_{m}$.

Proof. By Theorem 3.4 and Lemma 4.1, the map $\dot{a}_{m}: T B P J_{m} \rightarrow T B P J_{m}$ induces the map between the Adams towers, and hence we have a map $\alpha_{m}: Y J_{m} \rightarrow Y J_{m}$ such that $B P_{*}\left(\alpha_{m}\right)=a_{m}$.

q.e.d.

The condition on $m$ and $n$ indicates that the Adams-Novikov spectral sequence collapses, and so the cycle $a_{m}$ in $H^{0, *} E(n)_{*} /\left(J_{m}\right)$ survives to $Y J_{m_{*}}$. By Lemma 5.2, we have cofiber sequences

$$
Y J_{k} \stackrel{\alpha_{k}}{\rightarrow} Y J_{k} \stackrel{i_{k}}{\rightarrow} Y J_{k+1} \stackrel{j_{k}}{\rightarrow} \sum Y J_{k}
$$

for each $k<n$ with $E(n)_{*}\left(Y J_{k}\right)=E(n)_{*} /\left(J_{k}\right)$ and $E(n)_{*}\left(\alpha_{k}\right)=a_{k}$, which induces the exact sequence

$$
0 \rightarrow E(n)_{*} /\left(J_{k}\right) \stackrel{a_{k}}{\rightarrow} E(n)_{*} /\left(J_{k}\right) \stackrel{i_{k *}}{\rightarrow} E(n)_{*} /\left(J_{k+1}\right) \rightarrow 0 .
$$

Now recall [JMWZ] the Geometric Boundary Theorem. Let $E$ denotes a ring spectrum with $E_{*}(E)$ is flat over $E_{*}$. Then the $E$-Adams spectral sequence has the $E_{2}$-term

$$
E_{2}^{*, *}(X)=\operatorname{Ext}_{E_{*}^{*}(E)}^{*, *}\left(E_{*}, E_{*}(X)\right)
$$

The class $\bar{x} \in \mathbb{E}_{2}^{t, *}(X)$ is said to converge to $x \in \pi_{*}(X)$ provided that

(i) $\bar{x}$ is a permanent cycle representing the class $\{\bar{x}\} \in E_{\infty}^{t, *}(X)$;

(ii) $x \in F^{t} \pi_{*}(X)$ for the Adams filtration $F^{t}$; and

(iii) The homomorphism $F^{t} \pi_{*}(X) / F^{t+1} \pi_{*}(X) \rightarrow E_{\infty}^{t, *}(X)$ sends the $\operatorname{coset} x+F^{t+1} \pi_{*}(X)$ to $\{\bar{x}\}$.

(5.3) $\left[\mathbb{R} 2\right.$; Theorem 2.3.4] Let $\mathbb{E}$ be a ring spectrum with unit such that $\mathbb{E}_{*}$ is commutative and $E_{*}(E)$ is flat over $E_{*}$. Let $W \stackrel{f}{\rightarrow} X \stackrel{g}{\rightarrow} Y \stackrel{h}{\rightarrow} \Sigma W$ be $a$ 
cofiber sequence of finite spectra with $E_{*}(h)=0$. If $\bar{x} \in E_{2}^{t, *}(Y)$ converges to $x \in \pi_{*}(Y)$, then $\delta(\bar{x})$ converges to $h_{*}(x) \in \pi_{*}(W)$.

Here $\delta: E_{2}^{* \cdot *}(Y) \rightarrow E_{2}^{*, *}(W)$ is the connecting homomorphism of Ext groups. By [H; Lemma 6.2], we see that this theorem is applied to our case. For an invariant regular sequence $J_{m}$, we define a homotopy element $\alpha\left(J_{m}\right)$ by

$$
\alpha\left(J_{m}\right)=j_{0} j_{1} \cdots j_{m-2} a_{m-1} i_{m-2} \cdots i_{1} i_{0} \in \pi_{*}\left(L_{n} S^{0}\right) .
$$

Then by (5.3), we see that $g\left(J_{m}\right)$ converges to $\alpha\left(J_{m}\right) \in \pi_{*}\left(L_{n} S^{0}\right)$. In fact, $Y J_{0}=L_{n} S^{0}$ since the spectrum $\lim _{i}$ of $\infty$-Adams tower for $v_{n}^{-1} B P$ is homotopic to $L_{n} S^{0}$, which is shown by Hopkins and Ravenel.

Consider the localization map $\eta_{n}: S^{0} \rightarrow L_{n} S^{0}$. Then comparing the $E_{2}$-terms of the Adams-Novikov spectral sequences leads us to the statement that the map $\eta_{n *}: \pi_{*}\left(S^{0}\right) \rightarrow \pi_{*}\left(L_{n} S^{0}\right)$ sends the Greek letter elements to the Greek letter elements. For example, $\eta_{n *}\left(\alpha_{k}\right)=\alpha\left(p, v_{1}^{k}\right)$ and $\eta_{n *}\left(\beta_{k}\right)=\alpha\left(p, v_{1}, v_{2}^{k}\right)$. Furthermore, $\pi_{*}\left(L_{n} S^{0}\right)$ contains more such elements than $\pi_{*}\left(S^{0}\right)$ does. For example, it contains $\alpha\left(p, v_{1}, v_{2}, v_{3}, v_{4}^{k}\right)$ that may be called $\delta_{k}$.

\section{References}

[A] Adams, J. F., Stable homotopy and generalised homology, The University of Chicago Press, Chicago, 1974.

[B] Baas, N. A., On bordism theory of manifolds with singularities, Math. Scand., 33 (1973), 279-302.

[BP] Brown, E. H. and Peterson, F. P., A spectrum whose $\mathbb{Z}_{p}$ cohomology is the algebra of reduced $p$-th powers, Topology, 5 (1966), 149-154.

[H] Hikida, M., Relations between several Adams spectral sequences, Hiroshima Math. J., 19 (1989), 37-76.

[HS] Hopkins, M. J. and Smith, J. H., Nilpotence and stable homotopy theory II (to appear).

[JY] Johnson, D. C. and Yosimura, Z., Torsion in Brown-Peterson homology and Hurewicz homomorphisms, Osaka J. Math., 17 (1980), 117-136.

[L1] Landweber, P. S., Invariant regular ideals in Brown-Peterson homology, Duke Math. J., 42 (1975), 499-505

[L2] Homological properties of comodules over $M U_{*}(M U)$ and $B P_{*}(B P), A m e r$. J. Math., 98 (1976), 591-610.

[MRW] Miller, H. R., Ravenel, D. C. and Wilson, W. S., Periodic phenomena in the Adams-Novikov spectral sequence, Ann. of Math., 106 (1977), 469-516.

[P] Priddy, S., A cellular construction of BP and other irreducible spectra, Math. Z., 173 (1980), 29-34.

[R1] Ravenel, D. C., Localization with respect to certain periodic homology theories, Amer. J. Math., 106 (1984), 351-414.

[R2] - Complex cobordism and stable homotopy groups of spheres, Pure and Appl. 
Math., Academic Press, Inc., Orland, 1986.

[SY] Shimomura, K. and Yosimura, Z., $B P$-Hopf module spectrum and $B P_{*}$-Adams spectral sequence, Publ. RIMS, Kyoto Univ., 21 (1986), 925-947.

[T] Toda, H., On spectra realizing exterior part of the Steenrod algebra, Topology, 10 (1971), 53-65.

[W] Würgler, U., On products in a family of cohomology theories associated to the invariant prime ideals of $\pi_{*}(B P)$, Comment. Math. Helv., 52 (1977), 457-481. 